\title{
Screening for Melanoma in Men: a Cost-Effectiveness Analysis
}

\author{
Adewole S. Adamson, MD, MPP ${ }^{1,2}$, Jamie A. Jarmul, MD, $P h D^{3,4}$, and \\ Michael P. Pignone, MD, MPH ${ }^{1,2}$
}

'Department of Internal Medicine, Dell Medical School, University of Texas at Austin, Austin, TX, USA; ${ }^{2}$ LIVESTRONG Cancer Institutes, The University of Texas at Austin, Austin, TX, USA; ${ }^{3}$ University of North Carolina at Chapel Hill Department of Medicine, Chapel Hill, NC, USA; ${ }^{4}$ University of North Carolina-Chapel Hill Gillings School of Public Health, Chapel Hill, NC, USA.

\begin{abstract}
BACKGROUND: Systematic screening skin examination has been proposed to reduce melanoma-related mortality. OBJECTIVE: To assess the potential effectiveness of screening, in a demographic at high risk of melanoma mortality.

DESIGN: A cohort Markov state-transition model was developed comparing systematic screening versus usual care (no systematic screening). In the base case, we evaluated a sensitivity and specificity of $20 \%$ and $85 \%$, respectively, for usual care (incidental detection) and 50\% sensitivity and $85 \%$ specificity from systematic screening. We examined a wide range of values in sensitivity analyses.
\end{abstract}

PARTICIPANTS: Potential screening strategies applied to a hypothetical population of 10,000 white men from ages 50-75.

MAIN MEASURES: Incremental cost-effectiveness ratio, measured in cost per quality adjusted life year (QALY).

KEY RESULTS: Using base case assumptions, screening every 2 years beginning at age 60 reduced melanoma mortality by $20 \%$ with a cost-utility of $\$ 26,503$ per QALY gained. Screening every 2 years beginning at age 50 reduced mortality by 30\% with an incremental cost-utility of $\$ 67,970$ per QALY. Results were sensitive to differences in accuracy of systematic screening versus usual care, and costs of screening, but were generally insensitive to costs of biopsy or treatment.

CONCLUSIONS: Assuming moderate differences in accuracy with systematic screening versus usual care, screening for melanoma every 2 years starting at age 50 or 60 may be cost-effective in white men. Results are sensitive to degree of difference in sensitivity with screening compared to usual care. Better studies of the accuracy of systematic screening exams compared with usual care are required to determine whether a trial of screening should be undertaken.

KEY WORDS: melanoma; screening; Markov model; cost-effectiveness.

$\overline{\text { Prior Presentations Society for Medical Decision Making Fall Meeting, }}$ October 11 th, 2016, poster presentation.

Electronic supplementary material The online version of this article (https://doi.org/10.1007/s11606-019-05443-3) contains supplementary material, which is available to authorized users.

Received May 8, 2019

Accepted September 26, 2019

Published online November 8, 2019
$\mathrm{J}$ Gen Intern Med 35(4):1175-81

DOI: $10.1007 / \mathrm{s} 11606-019-05443-3$

(c) Society of General Internal Medicine 2019

\section{INTRODUCTION}

Approximately 10,000 Americans die annually from melanoma. ${ }^{1}$ According to Surveillance, Epidemiology, and End Result (SEER) data from the National Cancer Institute (NCI), the incidence of invasive melanoma has increased dramatically from 8 per 100,000 in 1975 to 25 per 100,000 in $2014 .^{2}$ Given that mortality has also increased over the same time period, efforts have been made to encourage population-based screening by clinicians, including primary care physicians, to improve early detection. ${ }^{3}$ In 2016, the US Preventive Services Task Force (USPSTF) concluded that there was insufficient evidence to assess the balance of benefits and harms of visual skin examination by a clinician to screen for melanoma in adults. ${ }^{4}$ However, the Agency for Healthcare Research and Quality (AHRQ) suggested future research focus on assessing the effectiveness of targeted screening higher risk patient populations. $^{5}$

While melanoma incidence has risen across demographic sub-groups, increases in mortality have been largely concentrated among white men over 65 years old, for whom mortality has increased from 9 to 24 per 100,000 between 1975 and $2014 .^{2}$ In contrast, melanoma mortality among white women over 65 years old has increased from 6 to 9 per 100,000 between 1975 and 2014. Given this disproportionate burden of melanoma-specific mortality among older white men, targeting this easily identifiable high-risk sub-group for screening may be a reasonable intervention strategy. For that reason, we sought to use modeling to study cost-effectiveness of melanoma screening in white men.

While a randomized trial is theoretically attractive for determining the effectiveness of periodic skin examinations, such a trial would require very large sample sizes to adequately power comparisons, and many years of follow-up, making it quite expensive. ${ }^{6}$ In the absence of large randomized controlled trials comparing screening strategies, clinical decision analysis and cost-effectiveness or cost-utility modeling can be used to explicitly compare alternative clinical options. 
Previous cost-effectiveness analyses of melanoma screening have suggested that screening could potentially be costeffective in certain high-risk groups such as those with a family history of melanoma. ${ }^{7}$ These studies, however, did not focus on the importance of the sensitivity and specificity of the screening visual skin examination. We define "systematic screening" as skin examination in the absence of skinrelated complaints by a primary care provider (PCP), compared with "usual care," defined as incidental diagnosis by a PCP or self-detection by a patient leading to a clinic visit.

In this analysis, we developed a Markov model to evaluate the cost-effectiveness of a set of skin cancer systematic screening strategies compared with usual care (no systematic screening) in a hypothetical population of 10,000 white men aged 50-75. Furthermore, we varied the sensitivity and specificity of the visual skin examination compared to usual care in order to evaluate the impact on the cost-effectiveness of different screening strategies.

\section{METHODS}

\section{Overview and Model Structure}

We developed a cohort state-transition Markov model to evaluate several screening strategies of different starting ages and intervals for screening, compared to usual care (Figure S1). A hypothetical population of 10,000 white men begins in the healthy state and then may transition every 12 months. We used annual cycles as they are commonly used and mimic annual visits for routine PCP visits. We modeled "undetected" and "detected" melanoma in situ, localized disease, regional disease, and metastatic disease as separate health states; costs, quality of life, and mortality rates differ in each state.

Our primary outcome measure was the incremental costeffectiveness ratio (ICER), measured in cost per quality adjusted life year (QALY). We assumed a willingness to pay (WTP) threshold of $\leq \$ 50,000$ per QALY in the base case, but we also examined other thresholds within the cost-effectiveness acceptability curves (CEAC). Total costs (in 2018 US dollars) and QALYs were calculated (discounting at 3\%/year for costs and utilities) over a lifetime horizon using a US health care system payer perspective, as recommended by the Second US Panel on Cost-Effectiveness. ${ }^{8}$ We also report clinical outcomes including melanoma mortality, incidence of melanoma by stage, and total cost of screening for each screening strategy. Total cost of screening was the sum of the cost of screening exams and biopsies for each strategy, less the sum of cost of biopsies in the base case (no screening) strategy.

\section{Melanoma Incidence and Rates of Progression}

We used age-adjusted melanoma incidence data from 2008 to 2012 SEER Medicare data for Caucasian men. ${ }^{8}$ We calibrated the transition probabilities for melanoma progression in the base model (no screening, i.e., usual care) to approximate data on age-adjusted melanoma mortality rate from 2008 to 2012 SEER Medicare data for Caucasian men, life expectancy after melanoma diagnosis, and melanoma stage distribution at diagnosis. ${ }^{9}$ More details of the model calibration are included in Table S2.

\section{Screening Strategies}

The median age of melanoma diagnosis is 63 years, and the median age at death is 69 years. Given that nearly $70 \%$ of melanomas are diagnosed after 55, we began our screening strategies at age 50 with intervals of 1,2 , or 5 years. ${ }^{9}$ We also modeled identical intervals beginning at age 60. Screening continued until patients reached age 75 .

\section{Test Characteristics for Usual Care vs. Skin Cancer Screening: Sensitivity and Specificity}

Because of the limited data on the accuracy of screening or usual detection, we modeled a wide range of test performance characteristics. In the base case "no screening" arm, we assumed a sensitivity and specificity of $20 \%$ and $85 \%$, respectively, for detection of any stage of melanoma, based on usual care and incidental detection; we assumed a sensitivity and specificity of $50 \%$ and $85 \%$, respectively, for systematic screening examinations. ${ }^{10-12}$ The sensitivity of usual care or screening determined the transition probabilities between the "undetected" and "detected" health states (Figure S1). The specificity determined the number of false positive screens, which we accounted for in the model by incurring cost of screening and biopsy while remaining in the "healthy" state.

\section{Cost and Utility Inputs}

Table 1 contains the base case estimates and ranges for our key parameters. Screening costs were determined by the average total allowed amount for a level 3 evaluation and management examination of a return Medicare patient. ${ }^{13}$ We assumed screening and biopsy costs of $\$ 73$ and $\$ 105$, respectively. ${ }^{13}$ Costs for treatment were determined based upon previously published literature, which report approximate costs by melanoma stage, as well as expert opinion. ${ }^{14,}{ }^{15}$ Cost estimates for stage 3 and 4 melanoma relied heavily on expert opinion, given that published data are not readily available in the era of immunotherapy and targeted therapy for melanoma. We therefore varied costs widely. We chose health state utilities based on previous estimates and expert opinion. ${ }^{14,16} \mathrm{We}$ assumed a decrement in quality of life after diagnosis of melanoma in situ because of the potential for anxiety related to diagnosis of cancer. ${ }^{17}$

\section{Deterministic and Probabilistic Sensitivity Analysis}

We conducted one- and two-way sensitivity analyses to examine the influence of key parameters. In the one-way analysis, we examined the effect of varying costs and health state utilities on preferred screening strategy. We used a two-way 
Table 1 Key Model Parameters

\begin{tabular}{|c|c|c|c|}
\hline Parameter & No Screening & Range & Source \\
\hline \multicolumn{4}{|l|}{ Utilities } \\
\hline Undetected MIS & 1 & - & Expert opinion, King 2011 \\
\hline Undetected local disease & 1 & _- & Expert opinion, King 2011 \\
\hline Undetected regional disease & 0.75 & $0.70-0.80$ & Tran 2018, King 2011 \\
\hline Undetected metastatic disease & 0.50 & $0.45-0.55$ & Tran 2018, King 2011 \\
\hline Detected MIS & 0.95 & $0.90-1.0$ & Tran 2018, King 2011 \\
\hline Detected local disease & 0.95 & $0.90-1.0$ & Tran 2018, King 2011 \\
\hline Detected regional disease & 0.75 & $0.70-0.80$ & Tran 2018, King 2011 \\
\hline Detected metastatic disease & 0.50 & $0.45-0.55$ & Tran 2018, King 2011 \\
\hline Healthy & 1 & - & \\
\hline \multicolumn{4}{|l|}{ Screening costs } \\
\hline Cost of screening exam & $\$ 73$ & $+/-20 \%$ & Medicare 2018 \\
\hline Cost of biopsy & $\$ 105$ & $+-20 \%$ & Medicare 2018 \\
\hline \multicolumn{4}{|c|}{ Treatment costs (applied for one cycle) } \\
\hline MIS & $\$ 1962$ & $+/-20 \%$ & Styperek 2012 \\
\hline Local disease & $\$ 12,932$ & $+-20 \%$ & Styperek 2012 \\
\hline Regional disease & $\$ 150,000$ & $+-50 \%$ & Expert opinion \\
\hline Metastatic disease & $\$ 250,000$ & $+-50 \%$ & Expert opinion \\
\hline \multicolumn{4}{|l|}{ Post-treatment costs (per year) } \\
\hline MIS & $\$ 73$ & $+/-20 \%$ & Medicare 2018 \\
\hline Local disease & $\$ 73$ & $+1-20 \%$ & Medicare 2018 \\
\hline Regional disease & $\$ 10,000$ & $+-50 \%$ & Expert opinion \\
\hline Metastatic disease & $\$ 10,000$ & $+-50 \%$ & Expert opinion \\
\hline \multicolumn{4}{|l|}{ Test characteristics for usual care } \\
\hline Sensitivity & $20 \%$ & $10-60 \%$ & Expert opinion \\
\hline Specificity & $85 \%$ & Not varied & Expert opinion \\
\hline \multicolumn{4}{|c|}{ Test characteristics for visual skin exam } \\
\hline Sensitivity & $50 \%$ & $30-90 \%$ & Chen 2001; Aitken 2006; Fritschi 2006 \\
\hline Specificity & $85 \%$ & Not varied & Aitken 2006; Fritschi 2006 \\
\hline
\end{tabular}

Melanoma in situ (MIS); all costs in USD 2018

analysis to show how preferred screening strategy changes when we varied sensitivity of usual care as well as sensitivity of the screening visual skin examination.

To represent the collective uncertainty in our analyses, we conducted a global probabilistic sensitivity analysis (PSA) using second-order Monte Carlo simulation ( $n=1000$ trials) to determine the effect of parameter uncertainty on the probability of cost-effectiveness (Crystal Ball, Oracle). The parameter ranges used in the PSA are reported in Table 1. We used gamma distributions to parameterize costs and beta distributions to parameterize health state utilities. ${ }^{18}$ We did not vary transition probabilities probabilistically given that we calibrated these parameters to observed melanoma mortality rate and stage distribution at diagnosis. Finally, we show CEACs to illustrate how the probability of cost-effectiveness changes with willingness-to-pay threshold. ${ }^{19}$ This study was exempt from IRB.

\section{RESULTS}

\section{Base Case}

Our main results are shown in Table 2. Screening every year starting at age 50 had the fewest deaths from melanoma. However, this screening strategy was also associated with the highest number of detected MIS cases, the highest total cost of skin cancer screening, and the highest total healthcare costs per individual.

Annual screening starting at age 50 was associated with the highest total QALYs (per individual) over a lifetime horizon, followed by annual screening starting at age 60. "No screening" had the lowest QALYs and lowest costs. Assuming a WTP of $\$ 50,000$ per QALY gained and our base case estimates of sensitivity of usual care and screening, the preferred screening strategy was screening every 2 years starting at age

Table 2 Model Outcomes over a Lifetime Horizon (50 years) for 10,000 50-Year-Old Men

\begin{tabular}{|c|c|c|c|c|c|c|c|}
\hline & \multirow[t]{2}{*}{ No Screening } & \multicolumn{2}{|c|}{$\begin{array}{l}\text { Annual screening, } \\
\text { starting at: }\end{array}$} & \multicolumn{2}{|c|}{$\begin{array}{l}\text { Biennial screening, } \\
\text { starting at: }\end{array}$} & \multicolumn{2}{|c|}{$\begin{array}{l}\text { Screen every } 5 \\
\text { years, starting at: }\end{array}$} \\
\hline & & 50 years & 60 years & 50 years & 60 years & 50 years & 60 years \\
\hline Total cost of screening & $\$ 0$ & $\$ 17.7 \mathrm{M}$ & $\$ 11.4 \mathrm{M}$ & $\$ 8.9 \mathrm{M}$ & $\$ 5.9 \mathrm{M}$ & $\$ 3.4 \mathrm{M}$ & $\$ 2.7 \mathrm{M}$ \\
\hline Number of detected MIS cases & 129 & 195 & 185 & 170 & 163 & 149 & 147 \\
\hline Number of detected local melanomas & 61 & 49 & 53 & 58 & 60 & 63 & 63 \\
\hline Number of detected regional melanomas & 15 & 9 & 10 & 12 & 13 & 16 & 16 \\
\hline Number of detected metastatic melanomas & 23 & 11 & 14 & 16 & 18 & 22 & 22 \\
\hline Number of melanoma deaths (detected and undetected) & 20 & 10 & 12 & 14 & 16 & 19 & 19 \\
\hline
\end{tabular}


Table 3 Base Case Incremental Cost-Effectiveness Ratios for Six Screening Strategies

\begin{tabular}{|c|c|c|c|c|c|}
\hline Screening strategy & QALYs/person & Costs/person & $\Delta$ Costs & $\triangle Q A L Y s$ & ICERs $(\Delta \operatorname{Costs} / \Delta Q A L Y s)$ \\
\hline Annual screening, starting at age 50 & 18.2993 & $\$ 1762$ & $\$ 478$ & 0.0041 & $\$ 116,640$ \\
\hline Annual screening, starting at age 60 & 18.2952 & $\$ 1284$ & - & - & WD* \\
\hline Screening every 2 years, starting age 50 & 18.2952 & $\$ 1279$ & $\$ 238$ & 0.0035 & $\$ 67,970$ \\
\hline Screening every 2 years, starting age 60 & 18.2917 & $\$ 1046$ & $\$ 109$ & 0.0041 & $\$ 26,503$ \\
\hline Screening every 5 years, starting age 50 & 18.2889 & $\$ 988$ & - & - & $\mathrm{WD}^{*}$ \\
\hline Screening every 5 years, starting at age 60 & 18.2876 & $\$ 937$ & $\$ 168$ & 0.0065 & $\$ 25,915$ \\
\hline No screening & 18.2811 & $\$ 769$ & - & - & - \\
\hline
\end{tabular}

*Weak dominance (WD): an alternate strategy is both more effective (higher QALYs) and has a lower ICER

60 , with an ICER of $\$ 26,503$ per QALY gained (Table 3). Screening every 2 years starting at age 50 had an incremental cost per QALY of $\$ 67,970$ and would be the preferred strategy using a WTP threshold of $\$ 100,000$ per QALY gained. Annual screening at 50 had an ICER of $\$ 116,640$ and would be preferred strategy at higher WTP thresholds.

\section{Deterministic Sensitivity Analysis}

When we varied individual parameters throughout the ranges listed in Table 1, we found that the preferred screening strategy was sensitive to costs of screening and metastatic melanoma treatment, but not sensitive to costs of individual screening exams, biopsy, melanoma treatment, nor to variation in health

Table 4 Effect of Varying the Accuracy of Screening and Usual Care on (A) Preferred Strategy at a WTP of \$50,000/QALY Gained and (B) Incremental Cost-Effectiveness of the Base Case Preferred Screening Strategy (Every 2 Years, Starting at Age 60)

\begin{tabular}{|c|c|c|c|c|c|c|c|}
\hline \multicolumn{2}{|c|}{ a } & \multicolumn{6}{|c|}{ Sensitivity of screening } \\
\hline & & $30 \%$ & $40 \%$ & $50 \%$ & $60 \%$ & $70 \%$ & $90 \%$ \\
\hline \multirow{5}{*}{ 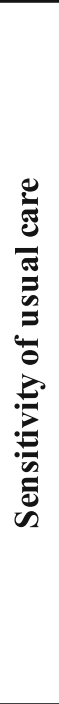 } & $10 \%$ & $\begin{array}{l}\text { Annual } \\
\text { screening, } \\
\text { starting at } \\
\text { age } 60\end{array}$ & $\begin{array}{l}\text { Annual } \\
\text { screening, } \\
\text { starting at } \\
\text { age } 60\end{array}$ & $\begin{array}{c}\text { Every } 2 \\
\text { years, } \\
\text { starting at } \\
50\end{array}$ & $\begin{array}{c}\text { Every } 2 \\
\text { years, } \\
\text { starting at } \\
50 \\
\end{array}$ & $\begin{array}{c}\text { Every } 2 \text { years, } \\
\text { starting at } 50\end{array}$ & $\begin{array}{c}\text { Every } 2 \text { years, } \\
\text { starting at } 50\end{array}$ \\
\hline & $20 \%$ & No screening & $\begin{array}{c}\text { Every } 2 \\
\text { years, } \\
\text { starting at } 60\end{array}$ & $\begin{array}{c}\text { Every } 2 \\
\text { years, } \\
\text { starting at } \\
60\end{array}$ & $\begin{array}{c}\text { Every } 2 \\
\text { years, } \\
\text { starting at } \\
60\end{array}$ & $\begin{array}{c}\text { Every } 2 \text { years, } \\
\text { starting at } 50\end{array}$ & $\begin{array}{c}\text { Every } 2 \text { years, } \\
\text { starting at } 50\end{array}$ \\
\hline & $30 \%$ & --- & No screening & $\begin{array}{c}\text { No } \\
\text { screening }\end{array}$ & $\begin{array}{c}\text { Every } 5 \\
\text { years, } \\
\text { starting at } \\
60\end{array}$ & $\begin{array}{c}\text { Every } 2 \text { years, } \\
\text { starting at } 60\end{array}$ & $\begin{array}{c}\text { Every } 2 \text { years, } \\
\text { starting at } 60\end{array}$ \\
\hline & $40 \%$ & --- & No screening & $\begin{array}{c}\text { No } \\
\text { screening }\end{array}$ & $\begin{array}{c}\text { No } \\
\text { screening }\end{array}$ & No screening & $\begin{array}{l}\text { Every } 5 \text { years, } \\
\text { starting at } 60\end{array}$ \\
\hline & $60 \%$ & --- & -- & --- & $\begin{array}{c}\text { No } \\
\text { screening }\end{array}$ & No screening & No screening \\
\hline \multirow{2}{*}{\multicolumn{2}{|c|}{ b }} & \multicolumn{6}{|c|}{ Sensitivity of screening } \\
\hline & & $30 \%$ & $40 \%$ & $50 \%$ & $60 \%$ & $70 \%$ & $90 \%$ \\
\hline \multirow{5}{*}{ 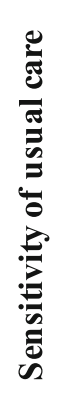 } & $10 \%$ & $\$ 27,884$ & $\$ 20,191$ & $\$ 15,756$ & $\$ 12,657$ & $\$ 10,255$ & WD* \\
\hline & $20 \%$ & $\$ 80,478$ & $\$ 41,029$ & $\$ 26,503$ & $\$ 18,789$ & $\$ 13,865$ & $\$ 7,225$ \\
\hline & $30 \%$ & --- & $\$ 144,684$ & $\$ 75,093$ & $\$ 51,500$ & $\$ 39,654$ & $\$ 37,247$ \\
\hline & $40 \%$ & --- & --- & $\$ 271,460$ & $\$ 147,030$ & $\$ 64,188$ & WD* \\
\hline & $60 \%$ & --- & --- & --- & -- & $\$ 1.31 \mathrm{M}$ & WD* \\
\hline
\end{tabular}

*Weak dominance (WD): an alternate strategy (screening every 2 years starting at age 50) is both more effective (higher QALYS) and has an ICER that is lower than the strategy shown in Table $4 B$ (screening every 2 years, starting at age 60) 


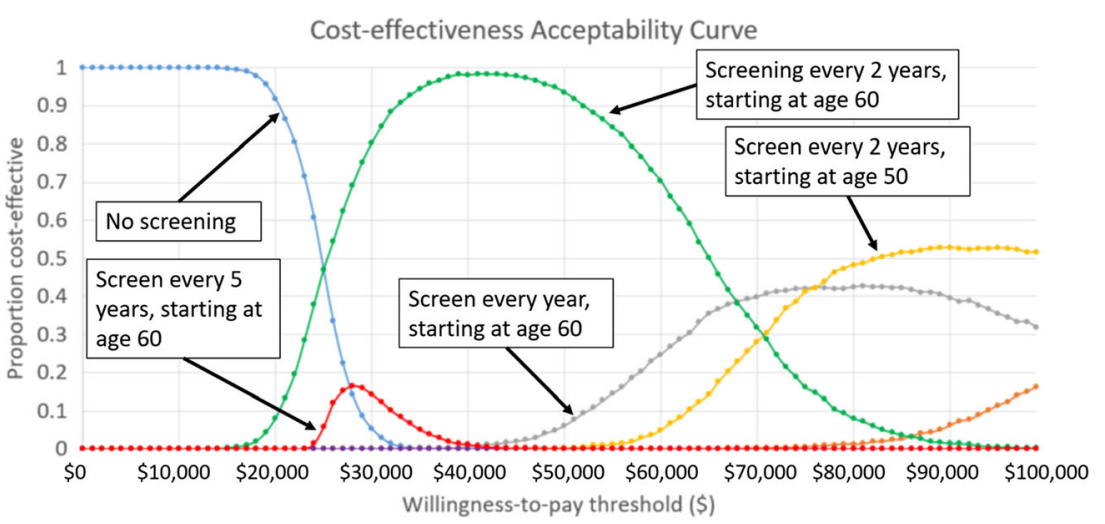

Figure 1 Cost-effectiveness acceptability curve using base case parameters. Proportion cost-effective represents the proportion of trials (out of 1000) for which each screening strategy was preferred at the corresponding willingness-to-pay threshold along the $x$-axis.

state utilities (Table S1). Notably, screening was no longer preferred at a screening cost greater than $\$ 146$, which was a 2fold increase in cost over our base case assumption of $\$ 73$ per screening exam. When the cost for metastatic melanoma treatment was increased to $\$ 750,000$ to account for the introduction of new immunotherapy treatments, ${ }^{20}$ we found that annual screening starting at age 60 was the preferred strategy.

Because of the importance of the differential sensitivity of usual care and screening, we performed two-way sensitivity analyses by varying the sensitivity of usual care (10-60\%) and the sensitivity of screening (30-90\%). We found that the ICERs and preferred screening strategies varied significantly with changes in the sensitivity of usual care vs. sensitivity of screening (Table 4).

Table 4 shows how the ICERs changed for our preferred screening strategy at WTP threshold of $\$ 50,000 /$ QALY gained of every 2 years starting at age 60 . For screening every 2 years starting at age 60 , we found that the ICER remained under $\$ 50,000 /$ QALY gained for two scenarios: (1) sensitivity of usual care $=10 \%$, sensitivity of screening ranging from 30 to $70 \%$; and (2) sensitivity of usual care $=20 \%$, sensitivity of screening ranging from 50 to $90 \%$.

Assuming a WTP of \$50,000/QALY gained, screening every 2 years starting at age 60 was the preferred strategy only for the base case scenario (Table 4). When sensitivity of screening improves, or sensitivity of usual care gets worse, the preferred strategy is to screen every 2 years starting at age 50. Furthermore, for screening to be cost-effective compared with usual care, we see that the difference in sensitivity of usual care vs. screening must be at approximately +30 percentage points or more, at least for cases where usual care is less than $40 \%$ (Table 4).

\section{Probabilistic Sensitivity Analysis}

At a WTP threshold of $\$ 50,000$ per QALY gained, the preferred screening strategy is to screen every 2 years starting at 60 , with an ICER of \$26,664 (95\% CI \$18,135-\$36,390) and a probability of cost-effectiveness of $94 \%$. However, both the preferred strategy and probability of cost-effectiveness vary dramatically by WTP threshold (Fig. 1). Using a WTP threshold of $\$ 25,000$ per QALY gained, no screening was the preferred strategy in $61 \%$ of simulations, with screening every 2 years starting at age 60 being the preferred strategy in $38 \%$ of simulations. At a WTP threshold of $\$ 100,000$ per QALY gained, the preferred strategy was to screen every 2 years, starting at age 50. However, this was the preferred strategy in only $52 \%$ of simulations; screening every year starting at age 50 was the preferred strategy in $32 \%$ of the simulations.

The CEACs change dramatically under different assumptions for sensitivity of usual care vs. screening. When both usual care and systematic screening have relatively high sensitivity for detecting melanoma (usual care 60\%; screening $90 \%$ ), we found that no screening is the preferred strategy for the entire range of tested WTP thresholds (CEAC not shown). However, under more favorable screening assumptions, no screening was preferred (Figure S2).

\section{DISCUSSION}

We found that screening every 2 years has the potential to reduce melanoma mortality by 20 to $30 \%$ in older white men aged 50-75 under reasonable assumptions about the accuracy of systematic screening examinations by clinicians compared with usual care. Given a willingness to pay of $\$ 50,000$ per quality adjusted life year gained, screening should begin at age 60 ; if society is willing to pay $\$ 100,000$ per life year gained, screening should begin at age 50. Cost-effectiveness was minimally dependent upon input costs of screening or melanoma treatment costs, but strongly dependent upon the assumptions made about the sensitivity of systematic screening compared with usual care, defined as no systematic screening (incidental detection).

Our results suggest that accurately quantifying the sensitivity of screening examinations compared with usual care is an important factor in determining the effectiveness and cost-effectiveness of screening. We consider "usual care" 
as melanoma incidentally detected by patients or by physicians during routine clinical exam, in the absence of systematic screening. Importantly, the higher the sensitivity of usual care, the less cost-effective systematic screening. Unfortunately, there are no high-quality studies comparing the sensitivity of screening examination to usual care. One fair quality cohort study performed in Queensland, Australia, assessed the effectiveness of skin cancer screening of clinicians and reported a $40.2 \%$ sensitivity and $86.1 \%$ specificity at 36 months of follow-up. ${ }^{11}$ However, this study did not measure usual care: clinicians were trained and encouraged to perform skin examinations to detect skin cancer. ${ }^{21}$ A separate Australian cohort study evaluated the effectiveness of melanoma screening by dermatologists or plastic surgeons and reported a sensitivity of $69.7 \%$ at 12 months, declining to $49 \%$ at 24 months of followup. Specificity in this study was $97.6 \% .^{12}$ While this study did measure sensitivity and specificity of screening among dermatologists and plastic surgeons, it did not measure the sensitivity and specificity of usual care. Importantly, while there are many studies of diagnostic accuracy which report sensitivity for the diagnosis of melanoma ranging between 42 to $100 \%$ among primary care clinician (PCP) and 81 to $100 \%$ among dermatologists, most of these studies involve the evaluation of images of individual pigmented lesions, which does not represent true screening or usual care. ${ }^{10}$ In practice few patients have full body skin exams, less than one third of primary care clinicians report screening their patients for skin cancer, and in a national survey of white middle-aged and older white men, only $15.7 \%$ reported having a skin exam in the past year. ${ }^{22,23}$

Previous cost-effective modeling studies have attempted to approximate the effects of screening versus no screening. ${ }^{24-26}$ One modeling study of adults above 20 years old estimated that a one-time skin cancer screening would cost $\$ 29,170$ per year of life saved compared to no screening. In men over 50 , the cost was $\$ 15,580 .^{26}$ The model was sensitive to the cost of screening, melanoma prevalence, and the probability of detecting localized disease; however, investigators did not vary the sensitivity or specificity of the screening examination, rather they assumed the difference in screen versus no screen as the difference in distribution between an America Academy of Dermatology screening program and SEER stage distributions. ${ }^{26}$, ${ }^{27}$ In an Australian study modeling screening for melanoma by PCPs, investigators varied the sensitivity of visual screening and found that at a sensitivity of $60 \%$, screening every 2 years for 20 years was cost-effective at $\$ 25,000$ per life year saved for men aged 50 years and $\$ 43,000$ per life year saved for women aged 50 years. ${ }^{25}$ However, this study did not adequately define the parameters of their usual care estimates. Lack of detailed evaluation of usual care is shortcoming of previous work modeling skin cancer screening strategies. Our study addresses this shortcoming by varying the sensitivity of usual care against the sensitivity of screening, which is a critical in the decision of whether or not to implement a screening program.
Our modeling has several strengths. Given the uncertainty about accuracy, we focused on the importance of sensitivity of systematic screening compared with usual care. Furthermore, in this analysis, we updated costs from previous studies and conducted multiple sensitivity analyses, including probabilistic analysis. ${ }^{24}$ As with previous studies, we found that melanoma screening could be costeffective in a high-risk group at a willingness to pay of $\$ 50,000$ per QALY. ${ }^{24-26}$

There are other risks associated with skin cancer screening that were not integrated into the model. Screening has potential associated harms including biopsy scarring, skin infections, and risk of overdiagnosis and overtreatment of cancers that may not be destined to progress. Overdiagnosis is of particular concern in melanoma in which incidence has quadrupled in the United States, and mortality across the population has not increased at a commensurate rate. $^{28,29}$ As part of skin cancer screening, it is also likely that incidental non-lethal, non-melanoma skin cancers (which are more common than melanoma) such as basal cell carcinoma and squamous cell carcinoma will be detected, resulting in an reduced morbidity, but an increase in cost. $^{30}$

\section{Limitations}

Our modeling study has limitations. Although the natural history data is based on the most current resources, modeling the behavior of melanoma and its relationship with screening involves uncertainty. Some melanomas may have a long radial growth phase, making them amenable to discovering during screening. However, some melanomas may have a rapid vertical growth phase causing them to quickly invade local tissues, making them less amenable to the benefits of screening. We attempted to mitigate this shortcoming by calibrating model transition probabilities to approximate age-adjusted melanoma mortality rate from SEER Medicare data for white men as well as published reports of stage distribution for melanoma at diagnosis. We limited the population in this study to middle-aged and older white men because we wanted to test the best case for screening in a high-risk group. Our findings may not be applicable to other groups such as younger men, women, and non-whites. Further modeling work will examine the cost-effectiveness in lower risk groups, such as women and non-white men.

\section{CONCLUSION}

Screening for melanoma every 2 years starting at age 50 or 60 may be cost-effective in white men. Results are sensitive to the degree of difference in sensitivity with screening compared to usual care. Future studies should consider a more accurate determination of the sensitivity and specificity of systematic screening for melanoma as compared to usual care. 
Contributors: Study concept and design: Adamson, Pignone

Acquisition, analysis, and interpretation of data: All Authors

Drafting of the manuscript: Adamson, Jarmul

Critical revision of the manuscript for important intellectual content: All Authors

Statistical analysis: Jarmul

Obtained funding: Adamson

Administrative, technical, or material support: Adamson

Study supervision: Pignone

IRB Statement: This study was exempt from IRB.

Corresponding Author: Adewole S. Adamson, MD, MPP; Department of Internal Medicine, Dell Medical School University of Texas at Austin, Austin, TX, USA (e-mail: adewole.adamson@austin.utexas. edu).

\section{Compliance with Ethical Standards:}

Conflict of Interest: Dr. Pignone is a former member of the US Preventive Services Task Force. The views expressed here are his and not necessarily those of the Task Force.

\section{REFERENCES}

1. Chen ST, Geller AC, Tsao H. Update on the Epidemiology of Melanoma. Curr Dermatol Rep. 2013;2(1):24-34.

2. SEER*Stat Database (1975-2016). Bethesda, MD: National Cancer Institute Surveillance Research Program, 2019 (http://www.seer.cancer.gov).

3. Ferris LK, Saul MI, Lin Y, et al. A Large Skin Cancer Screening Quality Initiative: Description and First-Year Outcomes. JAMA Oncol. 2017;3(8):1112-1115

4. Force USPST. Screening for skin cancer: Us preventive services task force recommendation statement. JAMA. 2016;316(4):429-435.

5. Wernli KJ, Henrikson NB, Morrison CC, Nguyen M, Pocobelli G, Blasi PR. Screening for Skin Cancer in Adults: Updated Evidence Report and Systematic Review for the US Preventive Services Task Force. JAMA. 2016;316(4):436-447.

6. Geller AC, Swetter SM, Oliveria S, Dusza S, Halpern AC. Reducing mortality in individuals at high risk for advanced melanoma through education and screening. J Am Acad Dermatol. 2011;65(5 Suppl 1):S87-94.

7. Gordon LG, Rowell D. Health system costs of skin cancer and costeffectiveness of skin cancer prevention and screening: a systematic review. Eur J Cancer Prev. 2015;24(2):141-149.

8. Sanders GD, Neumann PJ, Basu A, et al. Recommendations for Conduct, Methodological Practices, and Reporting of Cost-effectiveness Analyses: Second Panel on Cost-Effectiveness in Health and Medicine. JAMA. 2016;316(10): 1093-1103.

9. Howlader NNA, Krapcho M, Miller D, Bishop K, Altekruse SF, Kosary CL, Yu M, Ruhl J, Tatalovich Z, Mariotto A, Lewis DR, Chen HS, Feuer EJ, Cronin KA (eds). SEER Cancer Statistics Review. In. Bethesda, MD1975-2013.

10. Chen SC, Bravata DM, Weil E, Olkin I. A comparison of dermatologists and primary care physicians' accuracy in diagnosing melanoma: a systematic review. Arch Dermatol. 2001;137(12):1627-1634.

11. Aitken JF, Janda M, Elwood M, Youl PH, Ring IT, Lowe JB. Clinical outcomes from skin screening clinics within a community-based melanoma screening program. J Am Acad Dermatol. 2006;54(1):105-114.
12. Fritschi L, Dye SA, Katris P. Validity of melanoma diagnosis in a communitybased screening program. Am J Epidemiol. 2006;164(4):385-390.

13. Medicare Provider Utilization and Payment Data: Physician and Other Supplier https://www.cms.gov/Research-Statistics-Data-and-Systems / Statistics-Trends-and-Reports/Medicare-Provider-Charge-Data/Physician-and-Other-Supplier.html. Accessed February 6th, 2017.

14. Styperek A, Kimball AB. Malignant melanoma: The implications of cost for stakeholder innovation. Am J Pharm Ben. 2012;4(2):66-76.

15. Guy GP, Jr., Ekwueme DU, Tangka FK, Richardson LC. Melanoma treatment costs: a systematic review of the literature, 1990-2011. Am J Prev Med. 2012;43(5):537-545.

16. King SM, Bonaccorsi P, Bendeck S, et al. Melanoma quality of life: pilot study using utility measurements. Arch Dermatol. 2011;147(3):353-354.

17. Livingstone E, Krajewski C, Eigentler TK, et al. Prospective evaluation of follow-up in melanoma patients in Germany - results of a multicentre and longitudinal study. Eur J Cancer. 2015;51(5):653-667.

18. Briggs AH, Weinstein MC, Fenwick EA, et al. Model parameter estimation and uncertainty analysis: a report of the ISPOR-SMDM Modeling Good Research Practices Task Force Working Group-6. Med Decis Making. 2012;32(5):722-732.

19. Barton GR, Briggs AH, Fenwick EA. Optimal cost-effectiveness decisions: the role of the cost-effectiveness acceptability curve (CEAC), the cost-effectiveness acceptability frontier (CEAF), and the expected value of perfection information (EVPI). Value Health. 2008;11(5):886-897.

20. Oh A, Tran DM, McDowell LC, et al. Cost-Effectiveness of NivolumabIpilimumab Combination Therapy Compared with Monotherapy for FirstLine Treatment of Metastatic Melanoma in the United States. J Manag Care Spec Pharm. 2017;23(6):653-664.

21. Aitken JF, Elwood JM, Lowe JB, Firman DW, Balanda KP, Ring IT. A randomised trial of population screening for melanoma. J Med Screen. 2002;9(1):33-37.

22. Kirsner RS, Muhkerjee S, Federman DG. Skin cancer screening in primary care: prevalence and barriers. J Am Acad Dermatol. 1999;41(4):564-566

23. Coups EJ, Geller AC, Weinstock MA, Heckman CJ, Manne SL. Prevalence and correlates of skin cancer screening among middle-aged and older white adults in the United States. Am J Med. 2010;123(5):439445 .

24. Losina E, Walensky RP, Geller A, et al. Visual screening for malignant melanoma: a cost-effectiveness analysis. Arch Dermatol. 2007; 143(1):2128.

25. Girgis A, Clarke P, Burton RC, Sanson-Fisher RW. Screening for melanoma by primary health care physicians: a cost-effectiveness analysis. J Med Screen. 1996;3(1):47-53.

26. Freedberg KA, Geller AC, Miller DR, Lew RA, Koh HK. Screening for malignant melanoma: A cost-effectiveness analysis. J Am Acad Dermatol. 1999;41(5 Pt 1):738-745.

27. Koh HK, Norton LA, Geller AC, et al. Evaluation of the American Academy of Dermatology's National Skin Cancer Early Detection and Screening Program. J Am Acad Dermatol. 1996;34(6):971-978.

28. Siegel R, Naishadham D, Jemal A. Cancer statistics, 2013. CA Cancer J Clin. 2013;63(1):11-30.

29. Welch HG, Black WC. Overdiagnosis in cancer. J Natl Cancer Inst 2010;102(9):605-613.

30. Waldmann A, Nolte S, Geller AC, et al. Frequency of excisions and yields of malignant skin tumors in a population-based screening intervention of 360,288 whole-body examinations. Arch Dermatol. 2012;148(8):903-910

Publisher's Note Springer Nature remains neutral with regard to ju risdictional claims in published maps and institutional affiliations.? 